\title{
DESNUTRIÇÃO E SARCOPENIA EM IDOSOS RENAIS CRÔNICOS EM DIÁLISE PERITONEAL
}

\section{ARTIGO ORIGINAL}

BONHEUR, Licínio Rodrigues ${ }^{1}$, ALVES, Vicente Paulo ${ }^{2}$

BONHEUR, Licínio Rodrigues. ALVES, Vicente Paulo. Desnutrição e sarcopenia em idosos renais crônicos em diálise peritoneal. Revista Científica Multidisciplinar Núcleo do Conhecimento. Ano 06, Ed. 07, Vol. 01, pp. 05-35. Julho de 2021. ISSN: 2448-0959, Link de acesso: https://www.nucleodoconhecimento.com.br/saude/dialise-peritoneal, DOI: 10.32749/nucleodoconhecimento.com.br/saude/dialise-peritoneal

\section{RESUMO}

Introdução: A Doença Renal Crônica (DRC) e a idade avançada são fatores de risco para a sarcopenia e a desnutrição, associadas aos piores desfechos clínicos, incluindo a mortalidade. Problema: Qual a incidência percentual de desnutrição e sarcopenia em idosos renais crônicos em diálise peritoneal? Objetivo: $O$ objetivo deste trabalho foi avaliar a desnutrição e a sarcopenia em idosos portadores de doença renal crônica em diálise peritoneal. Métodos: Foram coletados dados sociodemográficos e clínicos, obtidos com quatro formulários por meio de entrevista presencial: GLIM, MAN, SARC$\mathrm{F}$ e formulário de coleta de dados clínicos e sociais, e antropometria e teste do dinamômetro portátil de 66 pacientes com idade igual ou superior a 60 anos, que realizavam diálise peritoneal há, pelo menos, 3 meses antes de iniciar o estudo, dividido em análise descritiva, de associação e de regressão logística. $O$ nível de significância utilizado em todo estudo foi de $p<0,05$. Resultados: A maioria dos pacientes encontravam-se sarcopênicos $(60,6 \%)$ e o resultado da GLIM foi desnutrição moderada ou grave $(55,4 \%)$. MAN triagem e MAN AEN com desnutridos ou em risco de desnutrição $(59,1 \%$ e $56,1 \%$, respectivamente). Houve associação

\footnotetext{
${ }^{1}$ Mestrando em Gerontologia.

2 Orientador.
} 
estatisticamente significativa para sarcopenia em relação às variáveis de sexo, resultado da GLIM e resultado das MANs triagem e AEN, e para desnutrição em relação às variáveis sexo, idade e albumina. As presenças de desnutrição e sarcopenia foram elevadas, como esperado, e confirmou-se a relação entre as duas doenças, e a associação entre a hipoalbuminemia e a desnutrição. Discussão: Verificou-se que o sexo feminino tem um risco maior de desenvolver desnutrição/sarcopenia. Não houve diferença estatisticamente significativa entre os grupos que faziam uso de suplementos nutricionais e realizavam atividades físicas. Mais estudos serão necessários, principalmente com delineamentos longitudinais, para se compreender os desfechos com intervenções nutricionais e educação física de maneira preventiva e não apenas na reabilitação.

Palavras-chave: Envelhecimento, Desnutrição, Sarcopenia, Doença renal crônica, Diálise peritoneal.

\section{INTRODUÇÃO}

Em virtude do envelhecimento da população, as doenças crônicas tornaram-se um problema de saúde pública, dentre elas, a Doença Renal Crônica (DRC) (BARBOSASILVA et al., 2016a). A faixa etária que mais cresce nos pacientes que fazem Terapia Renal Substitutiva (TRS) é a dos idosos (BARBOSA-SILVA et al., 2016b). A DRC cursa com importante depleção do estado nutricional dos pacientes, particularmente dessa faixa etária. A presença de desnutrição na DRC é conhecida, multifatorial e associada ao aumento da mortalidade e morbidade. O paciente em Diálise Peritoneal (DP) apresenta múltiplos fatores de risco para desnutrição energético-proteica (DEP), como alterações no apetite, anorexia, desconforto abdominal e absorção de glicose do dialisato (CRUZ-JENTOFT et al., 2019). Diante da gravidade e prognóstico reservado para os desnutridos em diálise, é necessário um controle rígido desses pacientes (HOLMES e SHOCKLEY, 2000). A sarcopenia é uma patologia muscular geriátrica que também está associada ao aumento da mortalidade e diminuição da qualidade de vida do idoso renal crônico. Ela se baseia em um tripé de redução da força muscular (critério de provável sarcopenia), associada à diminuição da massa muscular ou performance muscular (critérios confirmatórios) (JUSTINO, 2018). A 
International Society Peritoneal Dialysis (ISPD) sugere que haja avaliação nutricional de 6 a 8 semanas após o início da diálise peritoneal e acompanhamento nutricional a cada 4/6 meses. A avaliação nutricional deve ser minuciosa, envolvendo diversas metodologias, e assim investigando a desnutrição por meio da anamnese, dados antropométricos, dinamômetro, entre outras (CANTELMO, 2007). Desta forma, a questão norteadora para desenvolvimento deste estudo foi compreender: Qual a incidência percentual de desnutrição e sarcopenia em idosos renais crônicos em diálise peritoneal?

Sendo assim, o objetivo deste artigo foi avaliar a desnutrição e sarcopenia em idosos portadores de doença renal crônica em terapia renal substitutiva pelo método de diálise peritoneal. Tem, ainda, o propósito de verificar a sobreposição das entidades desnutrição e sarcopenia e de avaliar os fatores clínicos e sociais relacionados à desnutrição e à sarcopenia como sexo, idade, uso de suplemento, albumina, modalidade e tempo de tratamento, procedência do paciente, acompanhante, prática de atividade física e renda familiar

\section{MÉTODO}

Esse estudo foi descritivo e analítico, com delineamento transversal e cálculo estatístico. Como critério de inclusão foram considerados participantes com idade superior ou igual a 60 anos, e que realizaram diálise peritoneal (DP) por pelo menos três meses antes de iniciar o estudo.

Foram coletados dados sociodemográficos e clínicos, obtidos com 4 formulários por meio de entrevista presencial: GLIM (Anexo A), MAN (Anexo B), SARC-F (Anexo C) e formulário de coleta de dados clínicos e sociais (Apêndice B) e da antropometria e do teste do dinamômetro portátil.

O trabalho foi realizado em uma clínica de prevenção e tratamento de doenças renais (RenalCare), que se localiza no Distrito Federal, especializada em DP, que atende pacientes do Sistema Único de Saúde (SUS) e de convênio médico e particular, no período de julho a dezembro de 2019. A amostra total consistiu-se em 66 
participantes. O estudo foi dividido na análise descritiva, de associação e de regressão logística. As análises dos dados foram realizadas no programa IBM SPSS (Statistical Package for the Social Sciences, 23, 2015). O nível de significância utilizado em todo estudo foi de $p<0,05$.

\section{RESULTADOS}

As variáveis qualitativas foram apresentadas por meio da frequência (n) e porcentagem na Tabela 1. As figuras referentes à análise descritiva estão apresentadas no Anexo A deste relatório.

Observa-se que a maioria dos pacientes desse estudo eram do sexo feminino $(56,1 \%)$, realizando diálise peritoneal na modalidade DPA (diálise peritoneal automatizada) (89,4\%), não apresentavam DM (diabetes mellitus) (59,1\%), nem faziam uso de suplemento nutricional $(66,7 \%)$. A maior parte dos pacientes provém da hemodiálise (HD) (50,0\%), tinha acompanhante $(86,4 \%)$ e não praticava atividade física ou realizava fisioterapia $(84,8 \%)$. A maioria apresentou resultado da iniciativa de liderança global sobre desnutrição (GLIM - Global Leadership Initiative on Malnutrition) "bem nutrido" (44,6\%), com sarcopenia (60,6\%), resultado da Mini Avaliação Nutricional (MAN) triagem e Estado Nutricional Normal (MAN AEN) obtendo-se o resulta de 40,9 e $43,9 \%$ respectivamente.

Tabela 1. Caracterização sociodemográfica e clínica (variáveis qualitativas) de pacientes idosos realizando diálise peritoneal (DP) por pelo menos três meses na clínica de prevenção e tratamento de doenças renais (RenalCare). Brasília, de julho a dezembro de 2019.

\begin{tabular}{|l|l|l|}
\hline Variável & $\mathrm{n}$ & $\%$ \\
\hline Sexo & & \\
\hline Masculino & 29 & 43,9 \\
\hline Feminino & 37 & 56,1 \\
\hline
\end{tabular}




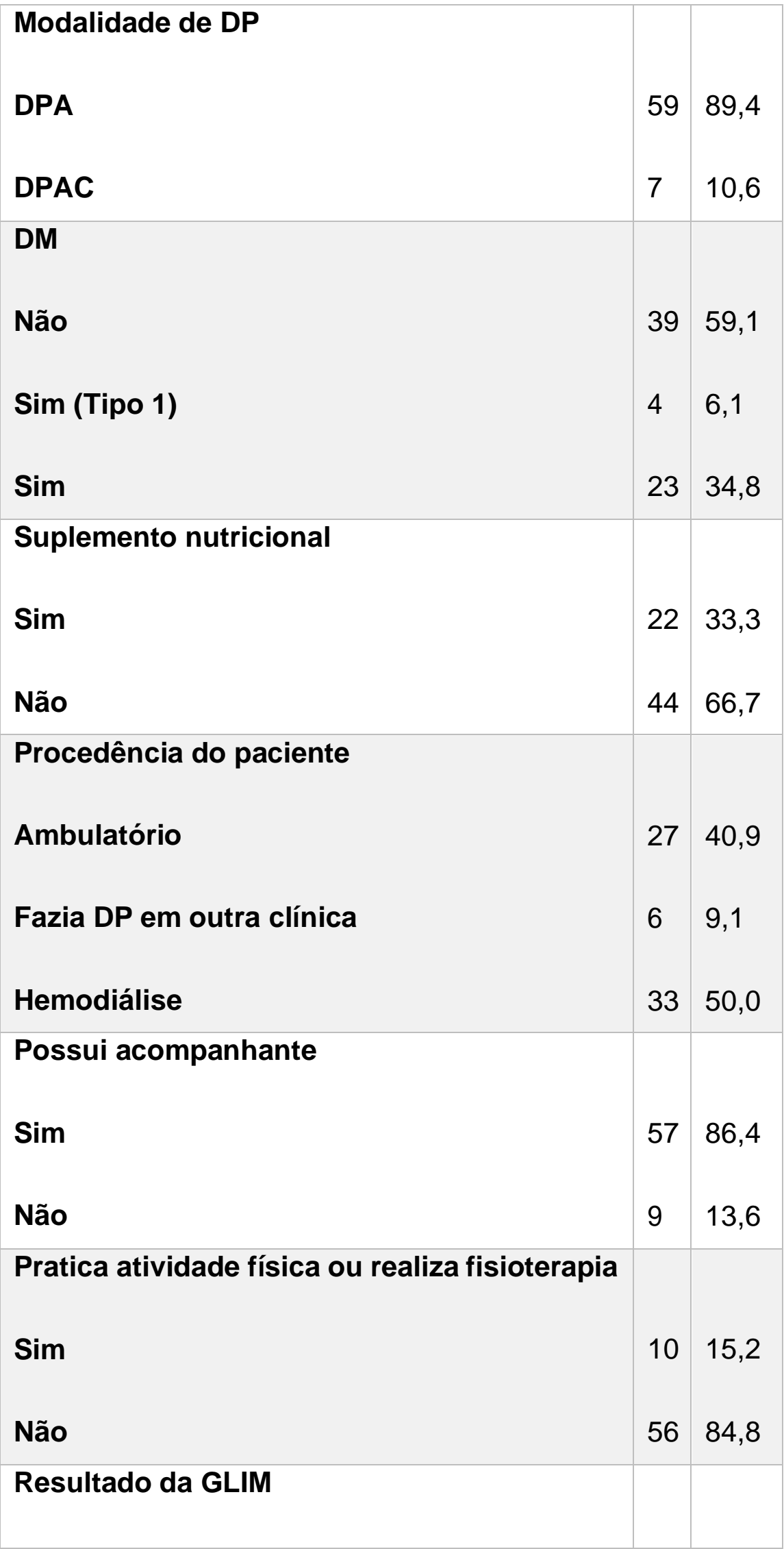




\begin{tabular}{|l|l|l|}
\hline Bem nutrido & 29 & 44,6 \\
\hline Desnutrição moderada & 28 & 43,1 \\
\hline Desnutrição grave & 8 & 12,3 \\
\hline Ausente & 1 & \\
\hline Sarcopenia & & \\
\hline Sim & 40 & 60,6 \\
\hline Não & 26 & 39,4 \\
\hline MAN triagem & & \\
\hline Desnutrido & 14 & 21,2 \\
\hline Sob risco de desnutrição & 25 & 37,9 \\
\hline Estado nutricional normal & 27 & 40,9 \\
\hline MAN AEN & & \\
\hline Desnutrido & 8 & 12,1 \\
\hline Sob risco de desnutrição & 29 & 43,9 \\
\hline Estado nutricional normal & 29 & 43,9 \\
\hline Total & 66 & 100,0 \\
\hline
\end{tabular}

Fonte: Autor

As variáveis quantitativas foram apresentadas por meio das medidas descritivas média, mediana, desvio padrão, mínimo, máximo e amplitude interquartil (Tabela 2). Os pacientes estudados apresentaram em média 70,86 anos, em tratamento há 2,61 anos em média, peso médio de 67,06 kg, altura média de 1,60 m, IMC médio de 26,04, CB (circunferência do braço) e CP (circunferência da panturrilha) médios de $31,58 \mathrm{~cm}$ e $34,45 \mathrm{~cm}$, respectivamente. A PCT (prega cutânea tríceps) média foi de 13,43 mm, 
a PCB (prega cutânea bíceps) média de 9,18 mm, albumina média de 3,56 g/dL, globulina média de $2,88 \mathrm{~g} / \mathrm{dL}$ e ferritina média de $438,91 \mathrm{ng} / \mathrm{mL}$. O valor médio do diagnóstico de sarcopenia (SARC-F) foi de 4,29\% com SARC-F + CP médio de 8,38. O resultado médio da MAN triagem foi de 10,23, o da MAN AEN, 22,09, o do dinamômetro foi de 21,09 em média e os pacientes apresentaram renda média de 3 mil reais.

Tabela 2. Caracterização sociodemográfica e clínica (variáveis quantitativas) de pacientes idosos realizando diálise peritoneal (DP) por pelo menos 3 meses na clínica de prevenção e tratamento de doenças renais (RenalCare); Brasília, de julho a dezembro de 2019.

\begin{tabular}{|l|l|l|l|l|l|l|l|}
\hline & $\mathrm{n}$ & Média & Mediana & Desvio & Mínimo & Máximo & Amplitude \\
\hline interquartil
\end{tabular}




\begin{tabular}{|l|l|l|l|l|l|l|l|} 
Dinamômetro & 66 & 21,09 & 20,00 & 9,49 & 4,00 & 42,00 & 16,00 \\
\hline $\begin{array}{l}\text { Renda mensal } \\
\text { (R\$) }\end{array}$ & 66 & 3181,27 & 2000,00 & 3053,73 & 750,00 & 15000,00 & 2125,00 \\
\hline
\end{tabular}

Fonte: Autor

\subsection{ANÁLISE DE ASSOCIAÇÃO}

A fim de avaliar qual seria o melhor teste para análise de associação das variáveis, foi utilizado o teste de Kolmogorov Smirnov (CANTELMO, 2007) para avaliar a normalidade das variáveis quantitativas. Para as variáveis idade, peso, IMC, CB, CP, globulina e dinamômetro não se rejeitou, em nível de significância de $p<0,05$, a normalidade dos dados, sendo, portanto, utilizados testes estatísticos paramétricos para elas e testes estatísticos não paramétricos para as demais.

Para as variáveis qualitativas, utilizou-se o teste Qui-quadrado de Pearson com correção de continuidade ou simulação e o teste de Monte Carlo (KAWAKAMI et al. 2015), quando necessário (esperavam uma frequência menor que 5). Para as variáveis quantitativas, utilizou-se o teste t de Student (com distribuição normal) e teste de Mann-Whitney (variáveis qualitativas ordinais e sem distribuição aproximadamente normal).

As variáveis deste trabalho foram associadas ao resultado de sarcopenia e aos resultados das avaliações nutricionais.

Houve associação estatisticamente significativa para sarcopenia em relação à variável sexo, resultado da GLIM e resultado das MANs triagem e AEN. Observa-se, na Tabela 3 , que pacientes do sexo feminino apresentaram significativamente mais sarcopenia em relação aos pacientes do sexo masculino ().

Tabela 3. Análise de associação entre Sarcopenia e Sexo de pacientes idosos realizando diálise peritoneal (DP) por pelo menos 3 meses na clínica de prevenção e tratamento de doenças renais (RenalCare), Brasília, de julho a dezembro de 2019. 


\begin{tabular}{|c|c|c|c|c|c|}
\hline & & & Sarco & & Total \\
\hline & & & Não & Sim & \\
\hline Sexo & Feminino & Contagem & 12 & 17 & 29 \\
\hline & & $\%$ & 30,0 & 65,4 & 43,9 \\
\hline & Masculino & Contagem & 28 & 9 & 37 \\
\hline & & $\%$ & 70,0 & 34,6 & 56,1 \\
\hline Total & & Contagem & 40 & 26 & 66 \\
\hline & & $\%$ & 100,0 & 100,0 & 100,0 \\
\hline
\end{tabular}

Fonte: Autor

Para o resultado da GLIM (Tabela 4), observa-se que pacientes com sarcopenia apresentaram significativamente mais tendência em ter desnutrição em relação aos pacientes sem sarcopenia (). Para os pacientes com desnutrição grave, observa-se que $87,5 \%$ apresentavam sarcopenia.

Tabela 4. Análise de associação entre Sarcopenia e Resultado da GLIM de pacientes idosos realizando diálise peritoneal (DP) por pelo menos 3 meses na clínica de prevenção e tratamento de doenças renais (RenalCare), Brasília, de julho a dezembro de 2019.

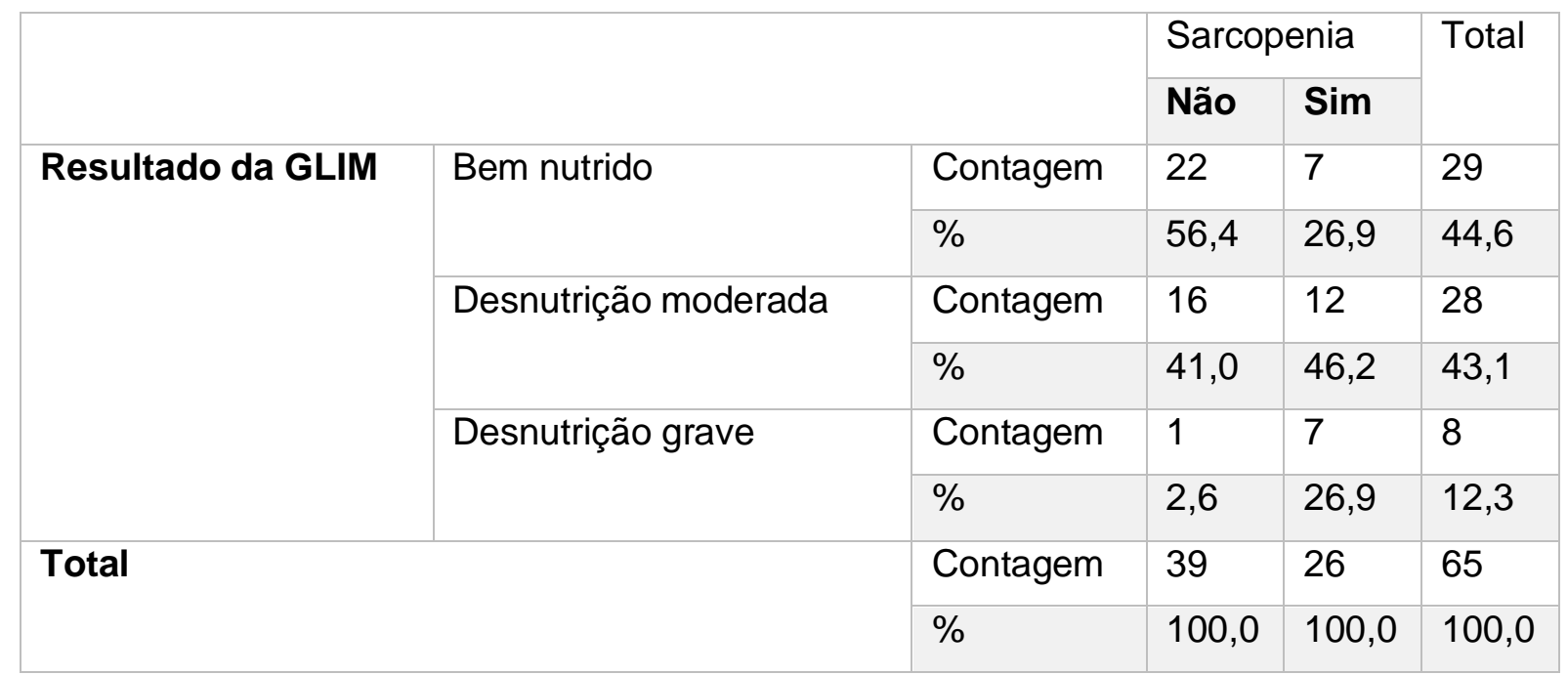

Fonte: Autor 
Em relação à MAN triagem (Tabela 5), pacientes desnutridos apresentaram significativamente mais sarcopenia em relação aos pacientes em outros estados nutricionais (). Para os pacientes desnutridos, observa-se que $71,4 \%$ apresentavam sarcopenia.

Tabela 5. Análise de associação entre Sarcopenia e MAN triagem de pacientes idosos realizando diálise peritoneal (DP) por pelo menos 3 meses na clínica de prevenção e tratamento de doenças renais (RenalCare), Brasília, de julho a dezembro de 2019.

\begin{tabular}{|c|c|c|c|c|c|}
\hline & \multicolumn{2}{|c|}{ Sarcopenia } & \multirow[t]{2}{*}{ Total } \\
\hline & & & Não & Sim & \\
\hline \multirow[t]{6}{*}{ MAN Triagem } & \multirow[t]{2}{*}{ Desnutrido } & Contagem & 4 & 10 & 14 \\
\hline & & $\%$ & 10,0 & 38,5 & 21,2 \\
\hline & \multirow[t]{2}{*}{ Sob risco de desnutrição } & Contagem & 16 & 9 & 25 \\
\hline & & $\%$ & 40,0 & 34,6 & 37,9 \\
\hline & \multirow[t]{2}{*}{ Estado nutricional normal } & Contagem & 20 & 7 & 27 \\
\hline & & $\%$ & 50,0 & 26,9 & 40,9 \\
\hline \multirow{2}{*}{\multicolumn{2}{|c|}{ Total }} & Contagem & 40 & 26 & 66 \\
\hline & & $\%$ & 100,0 & 100,0 & 100,0 \\
\hline
\end{tabular}

Fonte: Autor

Para a MAN AEN (Tabela 6), o mesmo resultado foi observado, onde pacientes desnutridos apresentaram significativamente mais sarcopenia em relação aos pacientes com outros estados nutricionais (). Para os pacientes desnutridos, observase que $75 \%$ apresentavam sarcopenia.

Tabela 6. Análise de associação entre Sarcopenia e MAN AEN de pacientes idosos realizando diálise peritoneal (DP) por pelo menos 3 meses na clínica de prevenção e tratamento de doenças renais (RenalCare), Brasília, de julho a dezembro de 2019.

\begin{tabular}{|l|l|l|l|l|l|}
\hline \multicolumn{2}{l}{} & \multicolumn{2}{|l|}{ Sarcopenia } & Total \\
\cline { 5 - 6 } MAN AEN & Desnutrido & Contagem & 2 & 6 & \\
\hline
\end{tabular}




\begin{tabular}{|l|l|l|l|l|l|}
\hline & & $\%$ & 5,0 & 23,1 & 12,1 \\
\cline { 2 - 7 } & $\begin{array}{l}\text { Sob risco de } \\
\text { desnutrição }\end{array}$ & Contagem & 15 & 14 & 29 \\
\cline { 2 - 7 } & $\begin{array}{l}\text { Estado nutricional } \\
\text { normal }\end{array}$ & Contagem & 23 & 6 & 29 \\
\cline { 3 - 7 } & & $\%$ & 57,5 & 23,1 & 43,9 \\
\hline Total & Contagem & 40 & 26 & 66 \\
\hline & & $\%$ & 100,0 & 100,0 & 100,0 \\
\hline
\end{tabular}

Fonte: Autor

Para as variáveis quantitativas, houve associação estatisticamente significativa entre sarcopenia e altura, MAN triagem, idade, peso, IMC, CB, CP, MAN AEN e dinamômetro. Observa-se, na Tabela 7, que os pacientes com sarcopenia apresentaram significativamente menores altura, valores de MAN triagem, peso, IMC, $\mathrm{CB}, \mathrm{CP}$, valores de MAN AEN e dinamômetro. Apenas a idade foi estatisticamente maior para pacientes com sarcopenia em relação aos pacientes sem sarcopenia

Tabela 7. Análise de associação entre Sarcopenia e variáveis quantitativas de pacientes idosos realizando diálise peritoneal (DP) por pelo menos 3 meses na clínica de prevenção e tratamento de doenças renais (RenalCare), Brasília, de julho a dezembro de 2019.

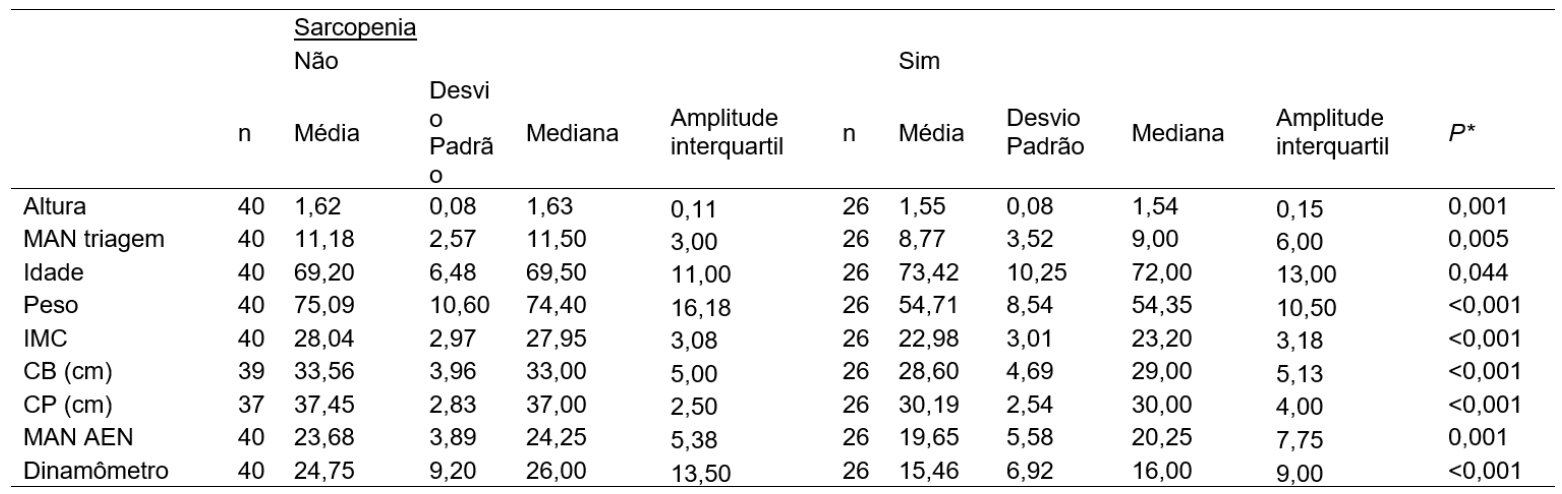

Fonte: Autor/ * teste T de Student ou teste de Mann Whitney.

As diversas variáveis estudadas também foram associadas aos estados nutricionais medidos pela GLIM, MAN (triagem) e MAN (AEN). Para as variáveis qualitativas, não houve associação estatisticamente significativa com os estados nutricionais. Já para

Disponível em: https://www.nucleodoconhecimento.com.br/saude/dialise-peritoneal 
as variáveis quantitativas, a associação foi significativa entre resultado da GLIM e PCB, albumina, ferritina, SARC-F, SARC-F + CP, MAN triagem, idade, peso, IMC, CB, CP, globulina, MAN AEN e dinamômetro. Os valores de PCB, albumina, peso, IMC, $\mathrm{CB}, \mathrm{CP}, \mathrm{MAN}$ triagem, MAN AEN e dinamômetro foram significativamente menores para os pacientes com desnutrição grave em relação aos demais. Já as variáveis ferritina, SARC-F, SARC-F + CP, idade e globulina foram significativamente maiores para os pacientes com desnutrição grave em relação aos demais (Tabela 8).

Tabela 8. Análise de associação entre Resultado da GLIM e variáveis quantitativas de pacientes idosos realizando diálise peritoneal (DP) por pelo menos 3 meses na clínica de prevenção e tratamento de doenças renais (RenalCare), Brasília, de julho a dezembro de 2019.

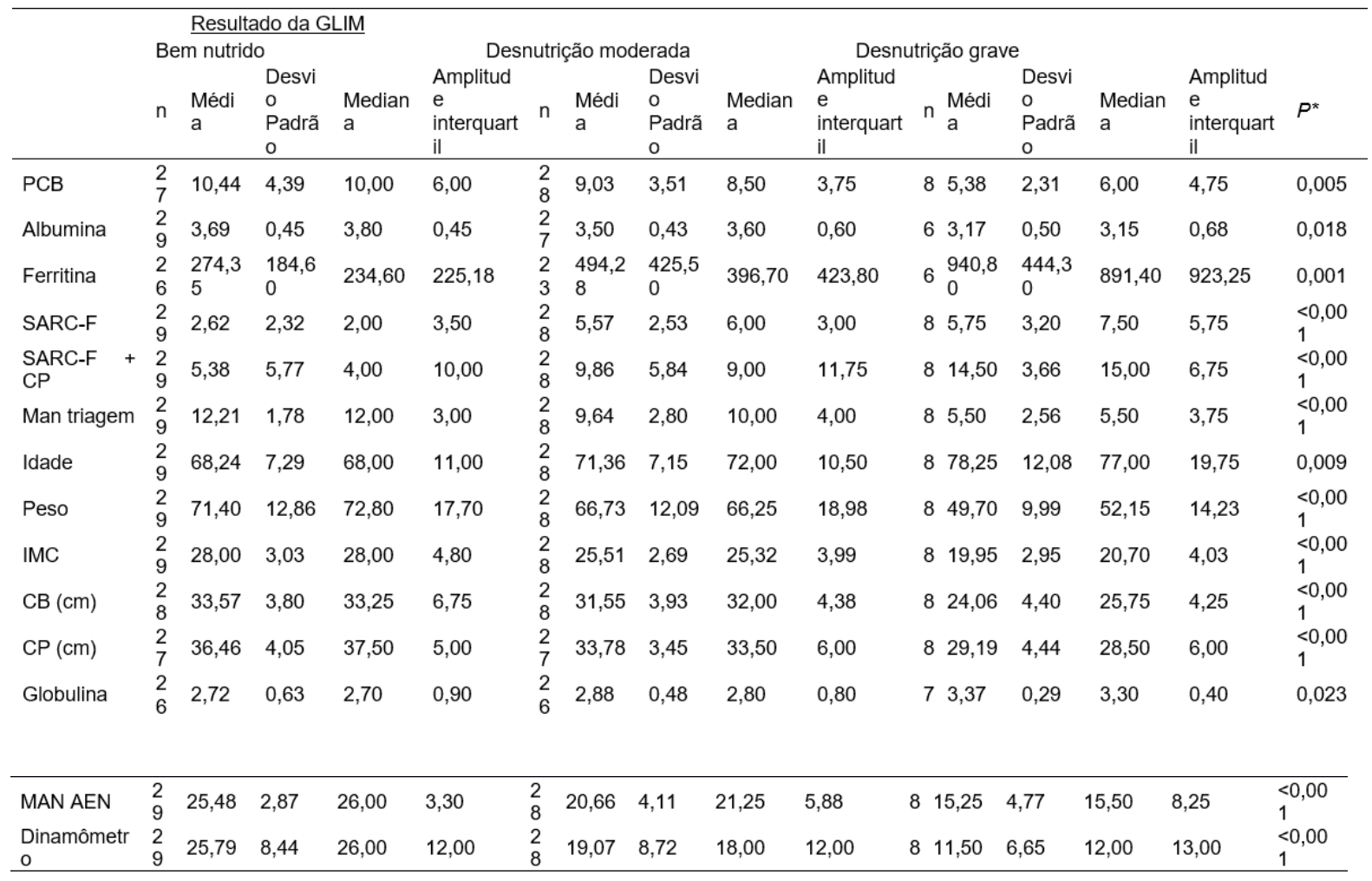

Fonte: Autor / * Análise de Variância unidirecional ou teste de Kruskal-Wallis.

Para o estado nutricional medido pela MAN (triagem), a associação foi significativa para as variáveis quantitativas PCB, SARC-F, SARC-F + CP, idade, IMC, CP e dinamômetro. 
Os valores de PCB, IMC, CP e dinamômetro foram significativamente menores para os pacientes desnutridos em relação aos demais. Já as variáveis SARC-F, SARC-F + $\mathrm{CP}$ e idade foram significativamente maiores para os pacientes desnutridos em relação aos demais (Tabela 9 ).

Em relação ao estado nutricional medido pela MAN (AEN), a associação foi significativa para as variáveis quantitativas de ferritina, SARC-F, SARC-F + CP, idade, peso, IMC, CB, CP e dinamômetro.

Os valores de peso, IMC, CB, CP e dinamômetro foram significativamente menores para os pacientes desnutridos em relação aos demais. Já as variáveis ferritina, SARCF, SARC-F + CP e idade foram significativamente maiores para os pacientes desnutridos em relação aos demais estados nutricionais (Tabela 10).

Tabela 9. Análise de associação entre Resultado da MAN (triagem) e variáveis quantitativas de pacientes idosos realizando diálise peritoneal (DP) por pelo menos 3 meses na clínica de prevenção e tratamento de doenças renais (RenalCare). Brasília, de julho a dezembro de 2019.

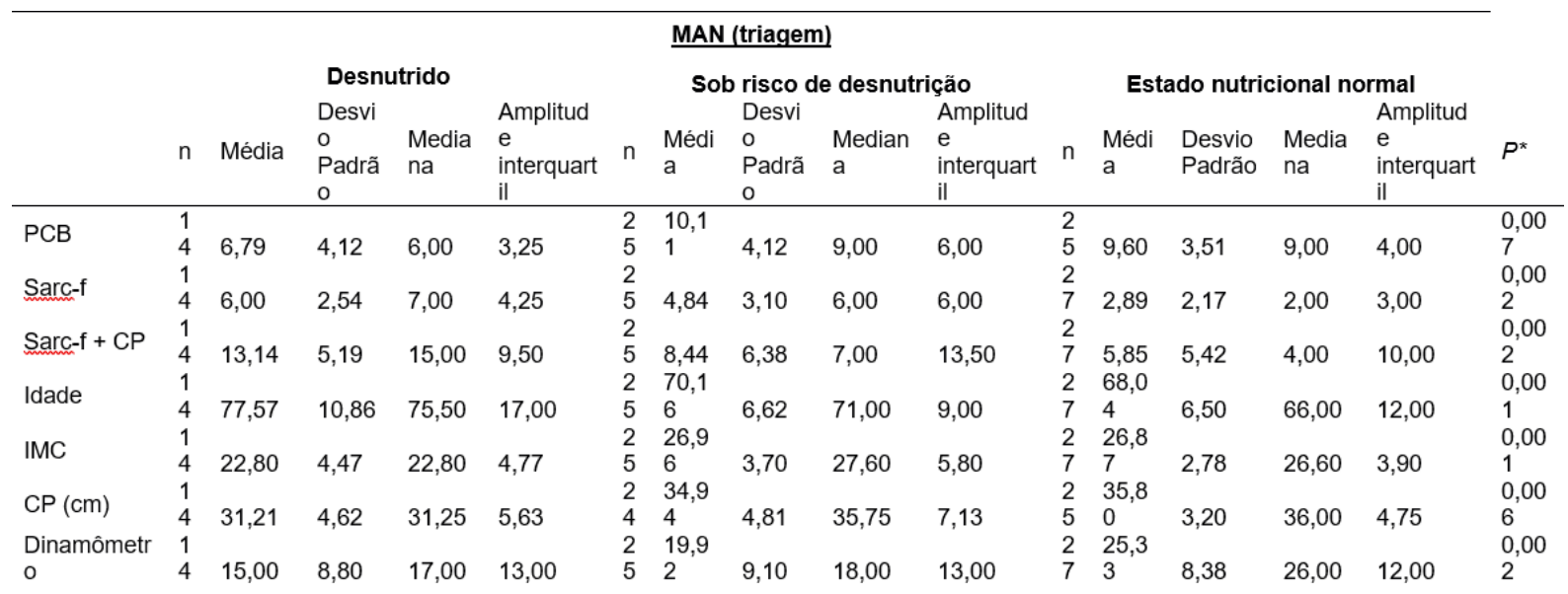

Fonte: Autor/ * Análise de Variância unidirecional ou teste de Kruskal-Wallis.

Tabela 10. Análise de associação entre Resultado da MAN (AEN) e variáveis quantitativas de pacientes idosos realizando diálise peritoneal (DP) por pelo menos 3 meses na clínica de prevenção e tratamento de doenças renais (RenalCare), Brasília, de julho a dezembro de 2019. 


\begin{tabular}{|c|c|c|c|c|c|c|c|c|c|c|c|c|c|c|c|}
\hline \multicolumn{16}{|c|}{ MAN (triagem) } \\
\hline & & \multicolumn{4}{|c|}{ Desnutrido } & \multicolumn{5}{|c|}{ Sob risco de desnutrição } & \multicolumn{5}{|c|}{ Estado nutricional normal } \\
\hline & $\mathrm{n}$ & Média & $\begin{array}{l}\text { Desvi } \\
\text { o } \\
\text { Padrã } \\
\text { o } \\
546.5\end{array}$ & $\begin{array}{l}\text { Media } \\
\text { na } \\
411.7\end{array}$ & $\begin{array}{l}\text { Amplitud } \\
\mathrm{e} \\
\text { interquart } \\
\text { il }\end{array}$ & 2 & $\begin{array}{l}\text { Média } \\
505.5\end{array}$ & $\begin{array}{l}\text { Desvi } \\
\text { o } \\
\text { Padrã } \\
\text { o } \\
393,1\end{array}$ & $\begin{array}{l}\text { Media } \\
\text { na } \\
424,3\end{array}$ & $\begin{array}{l}\text { Amplitud } \\
\mathrm{e} \\
\text { interquart } \\
\text { il }\end{array}$ & $\mathrm{n}$ & $\begin{array}{l}\text { Média } \\
309,0\end{array}$ & $\begin{array}{l}\text { Desvi } \\
\text { o } \\
\text { Padrã } \\
\text { o } \\
268.8\end{array}$ & $\begin{array}{l}\text { Media } \\
\text { na } \\
202.9\end{array}$ & $\begin{array}{l}\text { Amplitude } \\
\text { interquartil }\end{array}$ \\
\hline Ferritina & 8 & 628,71 & 4 & 5 & 1069,05 & $\begin{array}{l}4 \\
2\end{array}$ & 0 & 5 & 5 & 316,08 & $\begin{array}{l}4 \\
2\end{array}$ & 5 & 8 & 0 & 267,18 \\
\hline Sarc-f & 8 & 7,38 & 1,51 & 8,00 & 1,00 & $\begin{array}{l}9 \\
2\end{array}$ & 5,21 & 2,83 & 6,00 & 4,50 & $\begin{array}{l}9 \\
2\end{array}$ & 2,52 & 1,96 & 2,00 & 3,00 \\
\hline Sarc-f + CP & 8 & 14,88 & 5,36 & 17,50 & 7,00 & $\begin{array}{l}9 \\
2\end{array}$ & 10,03 & 5,83 & 10,00 & 10,50 & $\begin{array}{l}9 \\
2\end{array}$ & 4,93 & 4,88 & 4,00 & 7,00 \\
\hline Idade & 8 & 80,00 & 11,02 & 75,50 & 17,00 & $\begin{array}{l}9 \\
2\end{array}$ & 71,21 & 7,88 & 72,00 & 12,00 & $\begin{array}{l}9 \\
2\end{array}$ & 68,00 & 6,11 & 66,00 & 10,50 \\
\hline Peso & 8 & 58,74 & 12,88 & 59,75 & 13,03 & $\begin{array}{l}9 \\
2\end{array}$ & 64,41 & 14,96 & 61,60 & 22,30 & $\begin{array}{l}9 \\
2\end{array}$ & 72,00 & 11,73 & 70,80 & 18,90 \\
\hline IMC & 8 & 22,06 & 3,60 & 22,80 & 3,50 & $\begin{array}{l}9 \\
2\end{array}$ & 25,52 & 3,85 & 26,44 & 5,50 & $\begin{array}{l}9 \\
2\end{array}$ & 27,66 & 3,03 & 27,10 & 4,15 \\
\hline $\mathrm{CB}(\mathrm{cm})$ & 8 & 28,38 & 7,29 & 27,50 & 6,75 & $\begin{array}{l}9 \\
2\end{array}$ & 30,64 & 4,28 & 30,00 & 6,00 & $\begin{array}{l}8 \\
2\end{array}$ & 33,46 & 4,03 & 33,00 & 6,38 \\
\hline $\begin{array}{l}\mathrm{CP}(\mathrm{cm}) \\
\text { Dinamômetr }\end{array}$ & 8 & 29,75 & 3,96 & 30,50 & 6,25 & $\begin{array}{l}9 \\
2\end{array}$ & 33,62 & 3,90 & 34,50 & 7,00 & $\begin{array}{l}6 \\
2\end{array}$ & 36,83 & 3,88 & 37,25 & 5,00 \\
\hline 0 & 8 & 11,25 & 8,75 & 7,00 & 15,50 & 9 & 18,21 & 7,81 & 16,00 & 10,00 & 9 & 26,69 & 7,77 & 28,00 & 10,00 \\
\hline
\end{tabular}

Fonte: Autor $/{ }^{*}$ Análise de Variância unidirecional ou teste de Kruskal-Wallis.

\subsection{REGRESSÃO LOGÍSTICA}

Foram realizadas quatro regressões logísticas binárias múltiplas para identificar as principais variáveis que poderiam explicar os resultados da variável sarcopenia, GLIM, MAN (triagem) e MAN (AEN). Para sarcopenia, as categorias eram "sim" e "não", já para a variável GLIM, a divisão ficou em "desnutrição grave" e "demais", e para MAN (triagem e AEN), a divisão ficou em "pacientes desnutridos" e "demais".

O modelo matemático da regressão logística é apresentado a seguir:

$\operatorname{logit}[\pi(x)]=\beta_{0}+\beta_{1} x_{1}+\cdots+\beta_{n} x_{n}$

sendo: logit - função de ligação, $\pi(x)$ - probabilidade estimada, $x_{i}$ - variáveis independentes e $\beta_{i}$ - coeficientes estimados pela regressão logística.

Inicialmente, todas as variáveis foram inseridas no modelo, depois, foram utilizadas técnicas estatísticas de seleção de variáveis baseadas na inserção de variáveis (método forward stepwise: condicional, razão de verossimilhança e Wald) e na retirada de variáveis (método backward stepwise: condicional, razão de verossimilhança e Wald). As técnicas de retirada de variáveis apresentaram melhores ajustes e poder 
preditivo, sendo escolhido o método razão de verossimilhança por ser o mais adequado. A significância dos parâmetros da regressão foi testada pelo teste estatístico de Wald.

Para a variável resposta "'sarcopenia", inicialmente obteve-se o percentual de 61,9\% de concordância da tabela de classificação com o modelo apenas contendo o intercepto (constante). As variáveis independentes foram escolhidas de acordo com os principais fatores de risco relacionados a esses desfechos definidos no objetivo do estudo (sexo, idade, suplemento nutricional, albumina, modalidade de DP, tempo de tratamento, procedência do paciente, possui acompanhante, pratica atividade física ou fisioterapia e renda mensal). Ao inserir essas 10 variáveis independentes no modelo, o poder preditivo passou para $71,4 \%$, o que significa que o modelo prevê corretamente $71,4 \%$ dos casos de 'sarcopenia'. O $R^{2}$ de Nagelkerke foi de 0,286, indicando que $28,6 \%$ da variável sarcopenia foi adequadamente explicada pelo modelo com as 10 variáveis explicativas. O ajuste do modelo foi adequado: teste de Hosmer e Lemeshow com p-valor $=0,493$, ou seja, não se rejeita a hipótese nula de adequação do modelo. Apenas a variável "sexo" obteve coeficiente estatisticamente diferente de zero. Por isso, foram utilizadas técnicas de seleção de variáveis para melhorar o modelo matemático.

A técnica de seleção de variáveis comprovou o modelo com melhor ajuste após 9 etapas de exclusão de variáveis, mantendo apenas a variável "sexo" como significativa ao nível de significância de $5 \%$ (Tabela 11). O poder preditivo se manteve em $71,4 \%$, e o ajuste do modelo foi ótimo, com p-valor $=0,766$ para o teste de Hosmer e Lemeshow.

O valor da exponencial de $\beta$ representa a razão de chance $(R C)$ no modelo de regressão logística. Portanto, observa-se, ao mudar do sexo masculino para o feminino, um paciente aumentar em 3,517 vezes a chance de ter uma desnutrição grave.

Tabela 11. Análise de regressão logística para a variável resposta 'Sarcopenia' utilizando técnica de seleção de variáveis (backward stepwise) de pacientes idosos 
realizando diálise peritoneal (DP) por pelo menos 3 meses na clínica de prevenção e tratamento de doenças renais (RenalCare). Brasília, de julho a dezembro de 2019.

\begin{tabular}{|c|c|c|c|c|c|c|c|c|}
\hline \multirow[t]{2}{*}{$\begin{array}{l}\text { Variável } \\
\text { explicativa }\end{array}$} & \multirow[t]{2}{*}{ B } & \multirow[t]{2}{*}{ E.P. } & \multirow[t]{2}{*}{ Wald } & \multirow[t]{2}{*}{$\mathrm{gl}$} & \multirow[t]{2}{*}{$P$} & \multirow[t]{2}{*}{$\operatorname{Exp}(B)$} & \multicolumn{2}{|c|}{$\begin{array}{l}95 \% \text { C.I. para } \\
\operatorname{EXP(B)}\end{array}$} \\
\hline & & & & & & & Inferior & Superior \\
\hline Sexo & 1,26 & 0,56 & 5,01 & 1 & 0,025 & 3,517 & 1,169 & 10,5801 \\
\hline $\begin{array}{l}\text { Renda mensal } \\
(\mathrm{R} \$)\end{array}$ & 0,00 & 0,00 & 2,77 & 1 & 0,096 & 1,000 & 1,00 & 1,00 \\
\hline Constante & 2,04 & 0,94 & 4,69 & 1 & 0,030 & 7,728 & & \\
\hline
\end{tabular}

Fonte: Autor

Para a variável resposta "resultado da GLIM", inicialmente, obteve-se o percentual de 90,3\% de concordância da tabela de classificação com o modelo apenas contendo o intercepto (constante). As variáveis independentes foram as mesmas utilizadas para avaliar os fatores de risco relacionados à sarcopenia (sexo, idade, suplemento nutricional, albumina, modalidade de DP, tempo de tratamento, procedência do paciente, possui acompanhante, pratica atividade física ou fisioterapia e renda mensal). Ao inserir essas 10 variáveis independentes no modelo, o poder preditivo permaneceu em $90,3 \%$, o que significa que o modelo prevê corretamente $90,3 \%$ dos resultados da GLIM. O $R^{2}$ de Nagelkerke foi de 0,348 , indicando que $34,8 \%$ da variável resultado da GLIM foi adequadamente explicada pelo modelo com as 10 variáveis explicativas. O ajuste do modelo foi adequado: teste de Hosmer e Lemeshow com $\mathrm{p}$ valor $=0,651$, ou seja, não se rejeita a hipótese nula de adequação do modelo. Apenas a variável albumina foi significativa (com coeficiente estatisticamente diferente de zero), assim, foram utilizadas técnicas de seleção de variáveis para melhorar o modelo matemático.

A técnica de seleção de variáveis comprovou o modelo com melhor ajuste após nove etapas de exclusão de variáveis, mantendo apenas uma variável significativa em nível de significância de $5 \%$ (albumina) (Tabela 12), também mantendo o poder preditivo 
de $90,3 \%$ e adequado ajuste do modelo, com p-valor $=0,165$ para o teste de Hosmer e Lemeshow.

O valor da exponencial de $\beta$ representa a razão de chance (RC) no modelo de regressão logística. Portanto, observa-se que a cada diminuição em uma unidade na albumina (coeficiente negativo, ou seja, a diminuição da albumina está significativamente associada à presença de desnutrição), um paciente aumenta em $1 / 0,173=5,779$ vezes a chance de ter uma desnutrição grave

Tabela 12. Análise de regressão logística para a variável resposta 'Resultado da GLIM' utilizando técnica de seleção de variáveis (backward stepwise) de pacientes idosos realizando diálise peritoneal (DP) por pelo menos 3 meses na clínica de prevenção e tratamento de doenças renais (RenalCare), Brasília, de julho a dezembro de 2019.

\begin{tabular}{|c|c|c|c|c|c|c|c|c|}
\hline \multirow{2}{*}{$\begin{array}{l}\text { Variável } \\
\text { explicativa }\end{array}$} & \multirow[t]{2}{*}{ B } & \multirow[t]{2}{*}{ E.P. } & \multirow[t]{2}{*}{ Wald } & \multirow[t]{2}{*}{ gl } & \multirow[t]{2}{*}{$P$} & \multirow[t]{2}{*}{$\operatorname{Exp}(B)$} & \multicolumn{2}{|c|}{ 95\% C.I. para EXP(B) } \\
\hline & & & & & & & Inferior & Superior \\
\hline Albumina & $-1,75$ & 0,87 & 4,02 & 1 & 0,045 & 0,173 & 0,03 & 0,96 \\
\hline Constante & 3,72 & 2,87 & 1,68 & 1 & 0,195 & 41,449 & & \\
\hline
\end{tabular}

Fonte: Autor

Já para a variável resposta "MAN triagem", inicialmente, obteve-se o percentual de $81 \%$ de concordância da tabela de classificação com o modelo apenas contendo o intercepto (constante). As variáveis independentes foram as mesmas utilizadas para avaliar os fatores de risco relacionados à sarcopenia e resultado da GLIM (sexo, idade, suplemento nutricional, albumina, modalidade de DP, tempo de tratamento, procedência do paciente, possui acompanhante, pratica atividade física ou fisioterapia e renda mensal). Ao inserir essas 10 variáveis independentes no modelo, o poder preditivo passou para $85,7 \%$, o que significa que o modelo prevê corretamente $85,7 \%$ dos resultados de MAN triagem. $O \mathrm{R}^{2}$ de Nagelkerke foi de 0,361 , indicando que $36,1 \%$ da variável MAN triagem foi adequadamente explicada pelo modelo com as 10 variáveis explicativas. $\mathrm{O}$ ajuste do modelo foi adequado: teste de Hosmer e Lemeshow com $p$-valor $=0,398$, ou seja, não se rejeita a hipótese nula de adequação do modelo. 
Apenas a variável tempo de tratamento foi significativa e, sendo assim, foram utilizadas técnicas de seleção de variáveis para melhorar o modelo matemático.

A técnica de seleção de variáveis comprovou o modelo com melhor ajuste após 8 etapas de exclusão de variáveis, com 1 variável significativa ao nível de significância de $5 \%$ (idade) (Tabela 13), também com bom poder preditivo de $85,7 \%$, e bom ajuste do modelo, com $p$-valor $=0,214$ para o teste de Hosmer e Lemeshow.

O valor da exponencial de $\beta$ representa a razão de chance $(R C)$ no modelo de regressão logística. Portanto, observa-se que a cada aumento em 1 ano de idade, um paciente aumenta em 1,138 vezes a chance de apresentar desnutrição (avaliada pela MAN triagem).

Tabela 13. Análise de regressão logística para a variável resposta 'MAN triagem' utilizando técnica de seleção de variáveis (backward stepwise) de pacientes idosos realizando diálise peritoneal (DP) por pelo menos 3 meses na clínica de prevenção e tratamento de doenças renais (RenalCare). Brasília, de julho a dezembro de 2019.

\begin{tabular}{|c|c|c|c|c|c|c|c|c|}
\hline \multirow[t]{2}{*}{ Variável explicativa } & \multirow[t]{2}{*}{$\mathrm{B}$} & \multirow[t]{2}{*}{ E.P. } & \multirow[t]{2}{*}{ Wald } & \multirow[t]{2}{*}{ gl } & \multirow[t]{2}{*}{$P$} & \multirow[t]{2}{*}{$\operatorname{Exp}(B)$} & \multicolumn{2}{|c|}{$\begin{array}{l}95 \% \text { C.I. para } \\
\operatorname{EXP}(B)\end{array}$} \\
\hline & & & & & & & Inferior & Superior \\
\hline Idade & 0,13 & 0,05 & 5,84 & 1 & 0,016 & 1,138 & 1,02 & 1,26 \\
\hline Albumina & $-1,24$ & 0,75 & 2,76 & 1 & 0,097 & 0,290 & 0,07 & 1,25 \\
\hline $\begin{array}{l}\text { Tempo } \\
\text { tratamento }\end{array}$ & 0,39 & 0,21 & 3,45 & 1 & 0,063 & 1,480 & 0,98 & 2,24 \\
\hline Constante & $-7,55$ & 4,70 & 2,58 & 1 & 0,108 & 0,001 & & \\
\hline
\end{tabular}

Fonte: Autor

Por fim, para a variável resposta "MAN AEN", inicialmente, obteve-se o percentual de $88,9 \%$ de concordância da tabela de classificação com o modelo apenas contendo o intercepto (constante). As variáveis independentes foram as mesmas utilizadas para avaliar os fatores de risco das demais variáveis resposta (sexo, idade, suplemento nutricional, albumina, modalidade de DP, tempo de tratamento, procedência do 
paciente, possui acompanhante, pratica atividade física ou fisioterapia e renda mensal). Ao inserir essas 10 variáveis independentes no modelo, o poder preditivo passou para 93,7\%, o que significa que o modelo prevê corretamente $93,7 \%$ dos resultados de MAN AEN. O $R^{2}$ de Nagelkerke foi de 0,512 , indicando que $51,2 \%$ da variável MAN AEN foi adequadamente explicada pelo modelo com as 10 variáveis explicativas. O ajuste do modelo foi adequado: teste de Hosmer e Lemeshow com pvalor $=0,678$, ou seja, não se rejeita a hipótese nula de adequação do modelo. Porém nenhuma variável foi significativa e, com isso, também foram utilizadas técnicas de seleção de variáveis para melhorar e ajustar o modelo matemático.

A técnica de seleção de variáveis comprovou o modelo com melhor ajuste após nove etapas de exclusão de variáveis, com uma variável significativa ao nível de significância de 5\% (idade) (Tabela 14), ainda com bom poder preditivo, 92,1\%, e bom ajuste do modelo: $p$-valor $=0,430$ para o teste de Hosmer e Lemeshow.

O valor da exponencial de $\beta$ representa a razão de chance $(R C)$ no modelo de regressão logística. Portanto, observa-se que a cada aumento em 1 ano na idade, um paciente aumenta em 1,155 vezes a chance de ter desnutrição (avaliada pela MAN AEN).

Tabela 14. Análise de regressão logística para a variável resposta 'MAN AEN' utilizando técnica de seleção de variáveis (backward stepwise) de pacientes idosos realizando diálise peritoneal (DP) por pelo menos 3 meses na clínica de prevenção e tratamento de doenças renais (RenalCare). Brasília, de julho a dezembro de 2019.

\begin{tabular}{|c|c|c|c|c|c|c|c|c|}
\hline \multirow{2}{*}{$\begin{array}{l}\text { Variável } \\
\text { explicativa }\end{array}$} & \multirow[t]{2}{*}{$\mathrm{B}$} & \multirow[t]{2}{*}{ E.P. } & \multirow[t]{2}{*}{ Wald } & \multirow[t]{2}{*}{ gl } & \multirow[t]{2}{*}{$P$} & \multirow[t]{2}{*}{$\operatorname{Exp}(B)$} & \multicolumn{2}{|c|}{ 95\% C.I. para EXP(B) } \\
\hline & & & & & & & Inferior & Superior \\
\hline Idade & 0,14 & 0,07 & 4,91 & 1 & 0,027 & 1,155 & 1,02 & 1,31 \\
\hline Albumina & $-1,63$ & 0,88 & 3,41 & 1 & 0,065 & 0,196 & 0,03 & 1,11 \\
\hline Constante & $-7,07$ & 5,50 & 1,66 & 1 & 0,198 & 0,001 & & \\
\hline
\end{tabular}

Fonte: Autor 


\section{DISCUSSÕES}

Neste estudo, confirmou-se a alta prevalência de desnutridos e sarcopênicos em idosos em DP, da mesma forma que notamos em vários estudos (CRUZ-JENTOFT et al., 2019) (JUSTINO, 2018) (CANTELMO, 2007) (KAWAKAMl et al., 2015). De acordo com estudos de Kaysen (2000), em pacientes com doença renal crônica (DRC), a prevalência de sarcopenia varia de $5 \%$ a 37\%, dependendo da definição adotada e do estágio da doença renal. Em pacientes com DRC, independente do fator inicial, a perda de massa muscular depende de um balanço negativo da homeostase proteica. Além disso, a DRC está associada à regeneração prejudicada do tecido muscular (alterações estruturais das células, redução da ativação celular e inativação das células satélite) (LARSSON et al., 2019). Tanto a taxa de desnutrição, independentemente do método realizado, quanto a taxa de sarcopenia foram mais elevadas que as taxas encontradas na literatura, porém, essas taxas, em estudos anteriores, não foram restritas ao grupo alvo deste estudo, o que pode justificar esses valores elevados, principalmente na diferença encontrada na sarcopenia. Serão necessários mais estudos, preferencialmente longitudinais, para confirmar essa potencialização do risco de sarcopenia e desnutrição em pacientes idosos que realizam DP.

A literatura Lee et al. (2019) aponta que a fisiopatologia da sarcopenia na DRC é multifacetada e depende de: causas musculares, sistêmicas e comportamentais. A principal causa da perda muscular é a ativação do sistema ubiquitina-proteassoma (UPS). Tanto a inflamação (CRP, IL6 e TNFa) quanto a acidose metabólica desencadeiam a ativação do ATP-UPS. O TNFa desencadeia a degradação da proteína também ativando a via NFKB. A acidose metabólica ocorre principalmente através de um forte acúmulo da toxina urêmica indoxil sulfato (IS), que por um lado ativa o UPS e por outro induz estresse oxidativo e alterações metabólicas nas células musculares. Pacientes com DRC apresentam níveis elevados de angiotensina II, que ativa a via da caspase-3 e reduz o IGF-1 circulante e esquelético. Ambos os mecanismos estão associados à perda de proteína muscular e apoptose das células musculares (MARCKMANN, 1998). Tanto os níveis de insulina quanto a resistência à 
insulina podem contribuir para o desenvolvimento de sarcopenia em pacientes com DRC, uma vez que ambos os fatores ativam a via PI3K que está associada à perda de proteínas e à diminuição da síntese de proteínas (MEIRA et al., 2016).

Houve associação significativa do sexo feminino e da albumina com a desnutrição e sarcopenia. Pacientes do sexo feminino e/ou com valores mais baixos da albumina sérica devem ser acompanhados com maior frequência pelo risco elevado.

Curiosamente, não houve diferença significativa nos pacientes que utilizam complementos nutricionais ou que fazem atividade física. $\mathrm{O}$ pequeno $\mathrm{N}$ deve ser questionado fortemente por esses resultados. Além da pequena amostra, pode servir como explicação o fato de os pacientes estarem realizando apenas para reabilitação e não sendo prescritos de forma preventiva.

Em pacientes com DRC, a regeneração e o tamanho muscular também são afetados pelo acúmulo de miostatina, um regulador negativo da massa muscular esquelética (MIJNARENDS et al., 2016). Por fim, a sarcopenia também está associada à redução do apetite, inatividade física e comorbidades (PEREIRA et al., 2017). Na população em geral, a sarcopenia está associada a resultados negativos, como deficiência física, baixa qualidade de vida e mortalidade (REAL et al., 2018). Pacientes com DRC prédiálise afetados por sarcopenia têm um aumento geral de risco cardiovascular e mortalidade. Além disso, em pacientes com DRC, uma diminuição na força muscular está associada ao resultado composto de progressão para doença renal em estágio terminal e mortalidade (TAVARES et al., 2015).

O presente estudo confirmou a alta prevalência da desnutrição e sarcopenia nos pacientes idosos em terapia renal substitutiva. Valores tão elevados de entidades que cursam com desfechos clínicos negativos fazem sugerir que os agentes de saúde devam realizar um acompanhamento mais próximo com avaliação em intervalos mais curtos para a população idosa, tentando buscar um diagnóstico mais precoce e uma intervenção com maior eficácia. 


\section{CONSIDERAÇÕES FINAIS}

Este estudo partiu do questionamento sobre: Qual a incidência percentual de desnutrição e sarcopenia em idosos renais crônicos em diálise peritoneal? Desta feita, a partir do objetivo de avaliar a desnutrição e a sarcopenia nos idosos portadores de doença renal crônica em terapia renal substitutiva pelos métodos de diálise peritoneal. Foi triado a desnutrição, identificado a gravidade por meio do GLIM, avaliado a sarcopenia utilizando a ferramenta SARC-F + CP e verificado a sobreposição dessas entidades, a desnutrição e a sarcopenia em pacientes idosos.

Foi observado que o paciente idoso portador de doença renal crônica em diálise peritoneal tem grandes chances de desenvolver desnutrição e sarcopenia. Condições que aumentam a mortalidade e diminuem a qualidade de vida. Portanto, a avaliação periódica e de perto é mandatória para a prevenção e intervenção precoce pela equipe de saúde.

Entre as principais limitações impostas para a realização deste estudo foi a ausência de mais publicações a fim de correlacionar os resultados, e de pesquisas com exatamente o mesmo objetivo deste estudo. A maioria dos estudos compreendem pacientes em hemodiálise e não pacientes na modalidade de diálise peritoneal como o proposto aqui. Outro ponto também que pode ser considerado fator limitante foi o número pequeno da amostra.

Com relação a estudos futuros, deixa-se aqui sugestões para novos estudos sobre essa questão específica, com amostras maiores e que evidenciem formas terapêuticas de gestão desses casos e promoção à saúde e qualidade de vida. Devem-se estimular os estudos nesta área com enfoque na terapia dialítica peritoneal, método de terapia renal substitutiva tão subutilizado em nosso meio e carente de trabalhos. 


\section{REFERÊNCIAS}

BARBOSA-SILVA, G. T.; et al. Enhancing SARC-F: Improving Sarcopenia Screening in the Clinical Practice. Clinical Practice. Journal of the American Medical Directors Association, Hagerstown, v. 17, n. 12, p. 1136-1141, 2016a. doi: https://doi.org/10.1016/j.jamda.2016.08.004

BARBOSA-SILVA, G. T.; et al. Prevalence of sarcopenia among communitydwelling elderly of a medium-sized South American city: results of the COMO VAI? Study. Journal of cachexia, sarcopenia and muscle, Heidelberg, v. 7, n. 2, p. 136-143, 2016b. doi: 10.1002/jcsm.12049

CANTELMO, N. F. Desempenho de testes de normalidade multivariados avaliado por simulação Monte Carlo. Ciência e agrotecnologia, v.1, n 2, 2007

CRUZ-JENTOFT, A. J.; et al. Sarcopenia: revised European consensus on definition and diagnosis. Age and ageing, London, v. 48, n. 4, p. 16-31, 2019. doi: 10.1093/ageing/afy 169

HOLMES, C. J.; SHOCKLEY, T. R. Strategies to Reduce Glucose Exposure in Peritoneal Dialysis Patients. Peritoneal dialysis international: journal of the International Society for Peritoneal Dialysis. v. 20, Suppl 2, p. S37-S41, 2000.

JUSTINO, S. Consenso global sobre desnutrição - GLIM criteria for diagnosis of malnutrition - A consensus report from the global clinical nutrition community. Blog nutrição e saúde, 10 dez. 2018. Disponível em: https://prodiet.com.br/blog/2018/12/10/consenso-global-sobre-desnutricao-glimcriteria-for-diagnosis-of-malnutrition-a-consensus-report-from-the-global-clinicalnutrition-community/. Acesso em: 06 fev. 2021.

KAWAKAMI, R.; et al. Calf circumference as a surrogate marker of muscle mass for diagnosing sarcopenia in Japanese men and women. Geriatrics \& gerontology international, [s. I.], v. 15, n. 8, p. 969-976, 2015. https://doi.org/10.1111/ggi.12377 
KAYSEN, G. A. Malnutrition and the acute-phase reaction in dialysis patients how to measure and how to distinguish. Nephrology Dialysis Transplantation, [s. I.], v. 15, n. 10, p. 1521-1524, 2000. doi: https://doi.org/10.1093/ndt/15.10.1521

LARSSON, L.; et al. Sarcopenia: Aging-Related Loss of Muscle Mass and Function. Physiological Reviews, [s. I.], v. 99, n. 1, p. 427-511, 2019. doi: https://doi.org/10.1152/physrev.00061.2017

LEE, K.; et al. Recent Issues on Body Composition Imaging for Sarcopenia Evaluation. Korean journal of radiology, Seoul, v. 20, n. 2, p. 205-217, 2019. doi: https://doi.org/10.3348/kjr.2018.0479

MARCKMANN, P. Nutrition status of patients on hemodialysis and peritoneal dialysis. Clinical nephrology, Munchen-Deisenhofen, v. 29, n. 2, p. 75-78, 1998.

MEIRA, A. de S.; et al. Fragilidade em idosos com doença renal crônica em tratamento conservador. Revista Rene, Fortaleza, v. 17, n.3, p. 386-392, 2016.

MIJNARENDS, D. M.; et al. Physical activity and incidence of sarcopenia: the population-based AGES-Reykjavik Study. Age and ageing, London, v. 45. n. 5, p. 614-620, 2016. doi: https://doi.org/10.1093/ageing/afw090

PEREIRA, R. M.; et al. Qualidade de vida de idosos com doença renal crônica em tratamento conservador. Revista Brasileira de Enfermagem, v. 70, n. 4, p.851-859, 2017. doi: https://doi.org/10.1590/0034-7167-2017-0103

REAL, G. G.; et al. Calf Circumference: A Marker of Muscle Mass as a Predictor of Hospital Readmission. JPEN. Journal of parenteral and enteral nutrition, Thorofare, v. 42, n. 8, p. 1272-1279, 2018. doi: https://doi.org/10.1002/jpen.1170

TAVARES, E. L.; et al. Avaliação nutricional de idosos: desafios da atualidade. Revista Brasileira de Geriatria e Gerontologia, Rio de Janeiro, v. 18, n. 3, p. 643-650, 2015. doi: http://dx.doi.org/10.1590/1809-9823.2015.14249 


\section{APÊNDICE A - TERMO DE CONSENTIMENTO LIVRE E ESCLARECIDO}

O (a) Senhor(a) está sendo convidado(a) a participar do projeto: A avaliação da desnutrição e sarcopenia nos idosos em diálise peritoneal, sob responsabilidade do Prof. Dr. Vicente Paulo Alves e do mestrando em Gerontologia, médico nefrologista, Licinio Rodrigues Bonheur.

O objetivo desta pesquisa é avaliar a prevalência de desnutrição e sarcopenia nos idosos em diálise peritoneal. Esta pesquisa justifica-se, pois, o diagnóstico precoce e visualização dos pacientes em risco de nutrição podem ser prevenidos e tratados de forma mais rápida evitando desfechos clínicos desfavoráveis.

O(a) senhor(a) receberá todos os esclarecimentos necessários antes e no decorrer da pesquisa e the asseguramos que seu nome não aparecerá, sendo mantido o mais rigoroso sigilo por meio da omissão total de quaisquer informações que permitam identificá-lo(a). O(a) Senhor(a) pode se recusar a responder qualquer questão que the traga constrangimento, podendo desistir de participar da pesquisa em qualquer momento sem nenhum prejuízo.

A sua participação será da seguinte forma, a saber: ao chegar à clínica RenalCare para sua consulta mensal de rotina, responderá um questionário simples com cinco quesitos. Após o questionário, será submetido a coleta de dados através da pesagem, mensuração de altura e circunferência da panturrilha. $O$ tempo estimado para sua realização: 15 min.

Os resultados da pesquisa serão divulgados em trabalhos acadêmicos do Programa de Gerontologia da Universidade Católica de Brasília, podendo ser publicados posteriormente. Os dados e materiais utilizados na pesquisa ficarão sob a guarda do pesquisador.

Este projeto possui os seguintes benefícios para o paciente: avaliação nutricional com emissão dos resultados e explicação do padrão nutricional, além de orientações sobre 
dieta, suplementos, exercícios físicos e forneceremos uma cartilha de prevenção. Para a sociedade científica, servirá como um referencial teórico para aprimoramento e adequação do tratamento nos idosos em diálise peritoneal e apresenta os seguintes riscos, que são os considerados pequenos, porém que devem ser levados em consideração. Como risco do estudo existe o risco de queda durante a coleta dos dados que serão minimizados por meio do acompanhamento do idoso durante todo o tempo por um profissional. Para evitar o risco dos dados se tornarem públicos, todos os dados serão armazenados pelo pesquisador em um local confidencial, ao qual somente ele terá acesso.

É de nossa responsabilidade a assistência integral caso ocorra danos que estejam diretamente ou indiretamente relacionados à pesquisa. Esta pesquisa não Ihe trará custos e é de nossa responsabilidade o ressarcimento de custeio de despesas relacionadas à pesquisa.

Se o(a) Senhor(a) tiver qualquer dúvida em relação à pesquisa, por favor telefone para: Prof.

Vicente Paulo Alves, no Programa de Pós-Graduação da Universidade Católica de Brasília, telefone:

(61) 3356-9350, no horário comercial.

Este projeto foi aprovado pelo Comitê de Ética em Pesquisa da UCB, número do protocolo 3.782.438. As dúvidas com relação à assinatura do TCLE ou os direitos do sujeito da pesquisa podem ser obtidas no CEP/UCB pelo telefone: (61) 3356-9784. O CEP da UCB está localizado na sala K-239, no endereço Campus I - QS 07 - Lote 01 - EPCT - Águas Claras - Brasília - DF.

Este documento foi elaborado em duas vias, uma ficará com o pesquisador responsável e a outra com o voluntário da pesquisa.

Eu aceito participar da pesquisa: SIM ( ) NÃO ( ) 
Nome completo:

Assinatura:

Nome completo do pesquisador responsável:

Assinatura:

Brasília, _de de 2020

APÊNDICE B - FORMULÁRIO DE COLETA DE DADOS DA ANTROPOMETRIA 


\begin{tabular}{|l|}
\hline \multicolumn{1}{|c|}{ FORMULÁRIO DE COLETA DE DADOS DA ANTROPOMETRIA } \\
\hline IDENTIFICAÇÁO \\
Nome: \\
Número na pesquisa: \\
Idade: \\
Sexo: (1) Feminino ( ) Masculino \\
\hline DADOS ANTROPOMÉTRICOS \\
Peso: \\
Altura: \\
IMC: \\
Circunferência Abdominal: \\
Prega cutânea tríceps \\
(PCT): Prega cutânea \\
bíceps (PCB): \\
\hline DADOS FORÇA, CLíNICOS E SOCIAIS \\
Dados de força: Dinamômetro hidráulico \\
portátil F = Kgi \\
Dados Clínicos: Albumina sérica: \\
Dados Sociais: acompanhante ( ) Sim () não \\
Atividade física/fisioterapia ( ) sim ( ) não \\
Renda familiar: \\
\hline DADOS DO TRATAMENTO \\
Modalidade da diálise \\
peritoneal: () DPA ( ) \\
PDAC \\
Presença de Diabetes Melitus \\
Tempo de tratamento: \\
\hline
\end{tabular}

\section{ANEXO A - GLIM (INICIATIVA DE LIDERANÇA GLOBAL SOBRE DESNUTRIÇÃO)}




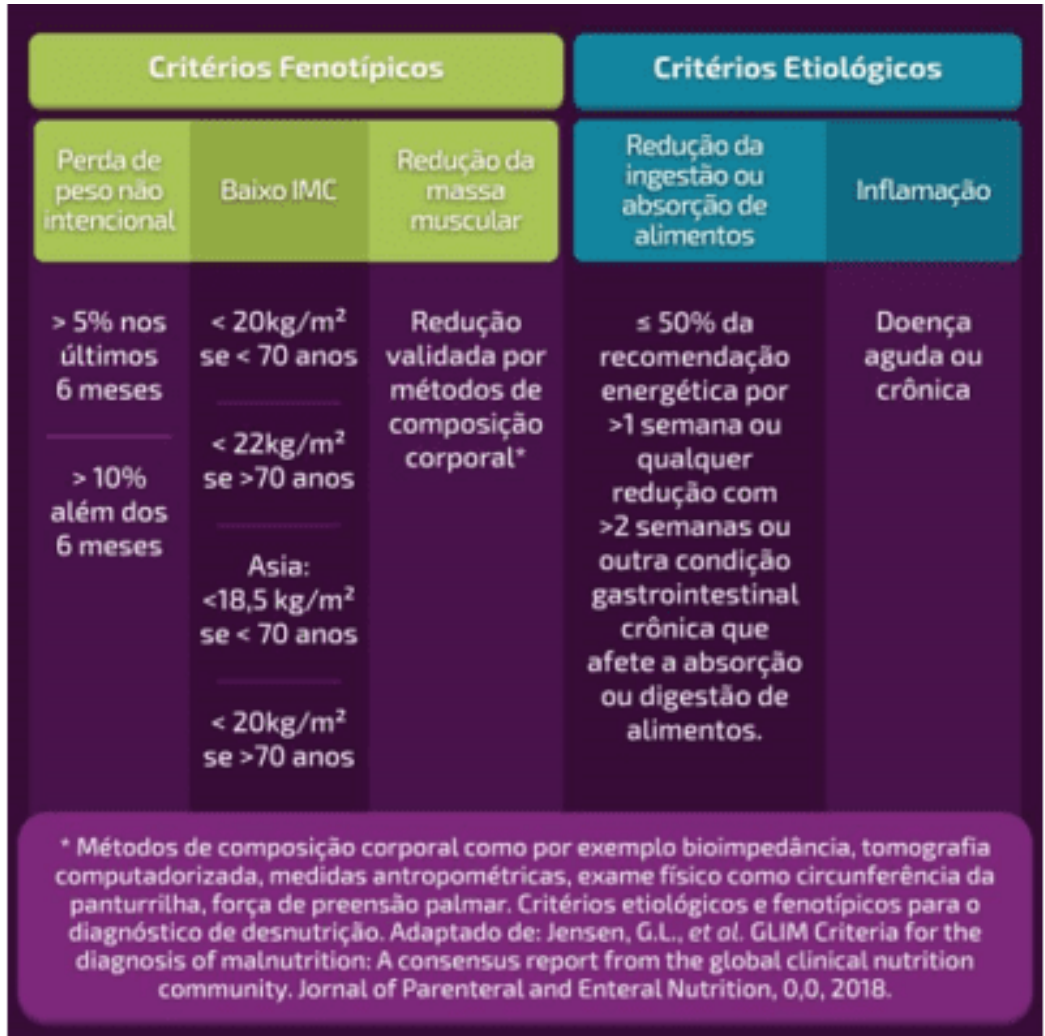

\begin{tabular}{|c|c|}
\hline \multicolumn{2}{|c|}{ CRITÉRIOS PARA MEDIR A GRAVIDADE DA DESNUTRIÇÃO } \\
\hline DESNUTRIÇĀOO MODERADA & DESNUTRIÇĀO GRAVE \\
\hline $\begin{array}{l}\text { Perda de } 5 \text { a } 10 \% \text { do peso habitual } \\
\text { nos últimos } 6 \text { meses }\end{array}$ & $\begin{array}{c}\text { Perda de > de } 10 \% \text { do peso habitual nos } \\
\text { últimos } 6 \text { meses }\end{array}$ \\
\hline ou & ou \\
\hline $\begin{array}{l}\text { Perda de } 10-20 \% \text { do peso habitual } \\
\text { em mais de } 6 \text { meses }\end{array}$ & $\begin{array}{c}\text { Perda de }>20 \% \text { do peso habitual em mais de } 6 \\
\text { meses }\end{array}$ \\
\hline ou & ou \\
\hline$I M C<20$ se tiver menos de 70 anos & $1 M C<18,5$ se tiver menos de 70 anos \\
\hline ou & ou \\
\hline IMC $<22$ se tiver 70 ou mais anos & $\mathrm{IMC}<20$ se tiver 70 ou mais anos \\
\hline ou & ou \\
\hline $\begin{array}{l}\text { Apresentar diminuição leve a moderada } \\
\text { da massa muscular esquelética, quantíficaçăo } \\
\text { feita usando um método com validade } \\
\text { comprovada }\end{array}$ & $\begin{array}{c}\text { Apresentar diminuição severa } \\
\text { da massa muscular esquelética, quantificaçăo } \\
\text { feita usando um método com validade } \\
\text { comprovada }\end{array}$ \\
\hline
\end{tabular}

\section{ANEXO B - MAN - MiniAvaliação Nutricional}




Apelibo:
\begin{tabular}{llll} 
Sexo: & Nome: & \\
\hline
\end{tabular}

Responda a seçaso triagem', preonchendo as caicas com os nümeros adequados. Some os nümeros da seçalo tragem".

Se a portusalo obtida for igual ou mence que 11, continue o preenchimento do questionario pera obter a pontuaçao indeadora de desrutriçso.

\section{Triagem}

A Nos ultimos trobs meses houve diminulcalo da ingesta alimentar devido a perda de apetine, problemas digestivos ou dificuldade para mastigar ou deglutir?

$0=$ diminukato grave da ingesta

1 = diminuicaso moderada da ingesta

2 = sem diminulgso da ingesta

B Perda de peso nos citimos 3 meses $0=$ seperior a trits qualas

$t=$ nalo rabe infomar.

2 = entre um e trets quilos

3 a sem perda de peso

C Mobllidade

0 = restrito ao leito ou a cadeira de rodas

1 in deambula mas nao e capaz de sar de cas

$2=$ nerma

D Passou por algum stress psicologico ou doença aguda nos Qintimos tods moses?

$0=\sin \quad 2=n a$

E Problemas neuropsicologicos

0 = demencia ou depressato graves

$i=$ demencia lgeira

2 = sem problemas psicolbojcos

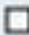

F Indice de Massa Corporal a pese em kg / (estatura em m) $0=B M C<19$

$1=19 \leq \mathrm{MMC}<21$

$2=21 \leq 1 \mathrm{MC}<2 \mathrm{2}$

$3=1 \mathrm{MC} \geq 2 \mathrm{2a}$

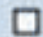

Pontuaçalo da Triagem (subtotal, maximo de 14 pontos)

12-14 ponlas: estado nutricional normal

g-11 ponios: sob risco de ceenuaricat

0.7 portics: desnutrido

Para uma avaliscalo mas detahadacontinue com as pergurtas $G-R$

\section{Avallaçăo global}

\section{G O doento vivo na sua própria casa}

(nso om institulcalo geniatrica ou hospital)

$1=\operatorname{sim} 20=n$

H Ueiliza mais de tris medicamentos diferentes por dia?

$$
0=\operatorname{sim} \quad 1=n a
$$

1 Lesces de pele ou escaras?
$0=\operatorname{sim} \quad 1=$ nas

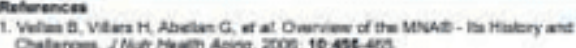

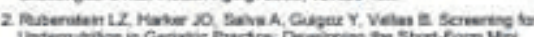

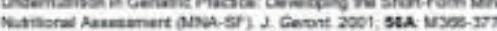

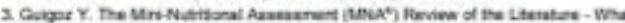

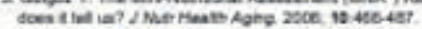

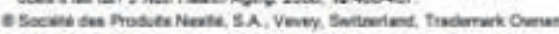



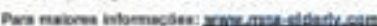

I Quantas refeicolos faz por dia?

$0=1$ uma refeigso

$1=$ duas releçotes

2 = tris refeipoes

K O doente consome:

- pela menos uma porçaso disria de kete

ou cerivados (ieite gaeplo icgurte)?

- guas ou mas porches semanais

de leguminesas ou cvos?

- came, peine 00 aves todos os dias?

$0.0=$ nenhuma ou uma resposta sims.

0.5 = das respostas 4 sim.

1.0 - bots respoctas esim.

1 o doente consome

ou produtios horticolas?

$0=$ nato $\quad 1=$ sim

M Ouantos copos de liquides (igua, sumo, caft, cha, leite) 0 doente consome por da?

D.0 5 menos de tris copos

$0.5=$ tres a cinco copos

$1.0=$ mais de chco copes

N Modo do se alimentar

0 = naso e capaz de se alimentar sazinho

$t$ = almenta se sozinho, portem com difculdade

2 = almenta-se sozinho sern dificuladode

O O doente acredita ter algum problema nuericional?

$0=$ acredies estar desnutrido

1 a naco sabe darer

2 = acredia nio ter um penbiema nutrioicnal

P. Em comparacso com outras pessoas da mesma lidade. como considora o doente a sua propria saúde?

$0.0=$ pior

$0.5=$ nato sice

$10=$ igual

$20=$ melher

Q Perimetro braquial (PB) em cm

$0.0=P B<21$

$0.5=21 \leq P B \leq 22$

$1.0=\mathrm{PB}>22$

$\sin \square$ nat $\square$

$\sin \square \operatorname{nac} \square$

$\sin \square \operatorname{cac} \square$

\section{R Perimetro da pema (PP) em cm}

$0=P P<31$

$1=P P \geq 31$

Avallaçso global (maxime 16 pontos)

Pontuaçalo da triagem

Pontuaçao total fmatrimo 30 pontos)

Arallisc to do Estado Nutricionat

de 24 a 30 pertos

de 17 a 23.5 pontos

menos de 17 portos

estado nutrioional norme

sob risco de desnutnçso desnutrido

\section{ANEXO C - SARC-F +CC}




\begin{tabular}{|c|c|c|}
\hline Componente & Pergunta & Pontuação \\
\hline Força & $\begin{array}{l}\text { O quanto de dificuldade você tem } \\
\text { para levantar e carregar } 5 \mathrm{~kg} \text { ? }\end{array}$ & $\begin{array}{l}\text { Nenhuma }=0 \\
\text { Alguma }=1 \\
\text { Muita, ou não consegue }=2\end{array}$ \\
\hline $\begin{array}{l}\text { Ajuda para } \\
\text { caminhar }\end{array}$ & $\begin{array}{l}\text { O quanto de dificuldade você tem } \\
\text { para atravessar um cômodo? }\end{array}$ & $\begin{array}{l}\text { Nenhuma }=0 \\
\text { Alguma }=1 \\
\text { Muita, usa apoios, ou incapaz }=2\end{array}$ \\
\hline $\begin{array}{l}\text { Levantar da } \\
\text { cadeira }\end{array}$ & $\begin{array}{c}\text { O quanto de dificuldade você tem } \\
\text { para levantar de uma cama ou } \\
\text { cadeira? }\end{array}$ & $\begin{array}{l}\text { Nenhuma }=0 \\
\text { Alguma }=1 \\
\text { Muita, ou não consegue sem ajuda }=2\end{array}$ \\
\hline Subir escadas & $\begin{array}{c}\text { O quanto de dificuldade você tem } \\
\text { para subir um lance de escadas de } 10 \\
\text { degraus? }\end{array}$ & $\begin{array}{l}\text { Nenhuma }=0 \\
\text { Alguma }=1 \\
\text { Muita, ou não consegue }=2 \\
\end{array}$ \\
\hline Quedas & $\begin{array}{l}\text { Quantas vezes você caiu no último } \\
\text { ano? }\end{array}$ & $\begin{array}{l}\text { Nenhuma }=0 \\
1-3 \text { quedas }=1 \\
4 \text { ou mais quedas }=2 \\
\end{array}$ \\
\hline Panturrilha & $\begin{array}{c}\text { Meça a circunferência da panturrilha } \\
\text { direita exposta do(a) paciente em pé, } \\
\text { com as pernas relaxadas e com os } \\
\text { pés afastados } \\
20 \mathrm{~cm} \text { um do outro }\end{array}$ & $\begin{array}{l}\text { Mulheres: } \\
\quad>33 \mathrm{~cm}=0 \\
\quad \leq 33 \mathrm{~cm}=10 \\
\text { Homens: } \\
>34 \mathrm{~cm}=0 \\
\leq 34 \mathrm{~cm}=10\end{array}$ \\
\hline $\begin{array}{l}\text { 0-10: sem sin: } \\
\text { 11-20: sugesti }\end{array}$ & $\begin{array}{r}\text { Somatório (0-20 } \\
\text { estivos de sarcopenia no momento ( } c \text { a } \\
\text { sarcopenia (prosseguir com investiga }\end{array}$ & $\begin{array}{l}\text { ontos) } \\
\text { gitar reavaliação periódica) } \\
\text { ão diagnóstica completa) }\end{array}$ \\
\hline
\end{tabular}

Enviado: Junho, 2021.

Aprovado: Julho, 2021. 